\title{
Characterizing Multi-Walled Carbon Nanotube Synthesis for Field Emission Applications
}

\author{
Benjamin L. Crossley ${ }^{1}$, Nathan E. Glauvitz¹, Betty T. Quinton², \\ Ronald A. Coutu ${ }^{1}$, Jr. and Peter J. Collins ${ }^{1}$ \\ ${ }^{1}$ Air Force Institute of Technology \\ ${ }^{2}$ Air Force Research Laboratory \\ USA
}

\section{Introduction}

The first evidence of carbon nanotubes comes from transmission electron microscope (TEM) micrographs published by Radushkevich in 1952 in the Russian Journal of Physical Chemistry (Radushkevich, 1952). However, without the capability to reliably produce, characterize, or use these carbon nanotubes little was done besides document their existence until 1991. Iijima effectively rediscovered or introduced carbon nanotubes to the scientific community as a by-product of an electric arc discharge method of synthesizing C60 fullerenes (Iijima, 1991). Since Iijima published his seminal article in Nature identifying multi-walled carbon nanotubes (MWCNT) in 1991, followed by the more significant discovery of single-walled carbon nanotubes (SWCNT) in 1993, research into the properties and applications of carbon nanotubes has flourished. In 1995, only four years after carbon nanotubes were introduced to the scientific community by Iijima, de Heer et al demonstrated the field emission capabilities of carbon nanotubes with the fabrication of a small electron gun using multi-walled carbon nanotubes (de Heer, 1995). CNTs have many unique physical and electrical properties making them ideal candidates for field emission sources. There are many potential applications for CNT based field emission devices ranging from flat panel displays to charge neutralization for electric propulsion on satellites. This research effort focuses on the growth of multi-walled carbon nanotubes for field emission. The potential applications being considered require that the CNT synthesis method be compatible with conventional substrate materials, chiefly silicon, and micro-frabrication processes to allow integration with conventional electronic devices. Of the many documented CNT synthesis methods, Chemical vapor deposition (CVD) synthesis occurs at low enough temperatures to facilitate silicon substrates. For this particular study two CVD processes, thermal chemical vapor deposition (T-CVD) and microwave plasma enhanced chemical vapor deposition (MPE-CVD), are used to produce MWCNT films or carpets.

The CNT growth process begins with substrate preparation. Each silicon substrate is first coated with a barrier layer of titanium or chrome. This thin film acts as an adhesion layer and as a diffusion barrier. It also facilitates the cathode connection to the CNTs during field emission tests. CNT growth via any CVD method requires a catalyst for the carbon atoms to nucleate around. The transition metals $\mathrm{Fe}, \mathrm{Ni}, \mathrm{Co}$, and alloys composed of one or more of these elements have proven effective as catalyst materials. For this effort, only 
sputtered $\mathrm{Ni}$ and $\mathrm{Fe}$ are considered for catalyst layers. The catalyst layer must be granulated into nanoparticles to facilitate CNT synthesis. The catalyst is broken into nanoparticles by high temperature annealing treatment in the T-CVD process and by a hydrogen plasma treatment in the MPE-CVD process. Both methods are carried out in situ and are followed immediately by the CNT synthesis process. The catalyst material, barrier layer, granulation and synthesis processes are varied and the resulting CNTs are tested for field emission.

Characterizing each step of the synthesis process and comparing the field emission capabilities of the resulting CNTs as the parameters are changed leads to a fabrication process optimized for field emission applications. The first step in characterizing each synthesis process is using scanning electron microscopy (SEM) to determine the size and spacing of the granulated nanoparticles after the annealing or hydrogen plasma pretreatment of the catalyst material. After CNT synthesis, the carbon nanotubes are characterized physically via Raman spectroscopy and SEM imagery. Raman spectroscopy provides a method to determine the quality of the CNTs. SEM micrographs are used to determine CNT areal density, presence of amorphous carbon, and CNT physical characteristics such as diameter, length, and alignment.

Next the field emission properties of the CNTs are characterized to include turn-on electric field strength, maximum current density at a fixed electric field strength, current density versus electric field strength relationship, and Fowler-Nordheim plot. Field emission tests are performed in a diode configuration under high vacuum. The CNTs are grounded and act as the cathode. The anode is a copper collector plate located above the CNTs. The anode is swept across a positive voltage range. The field emission current is measured through the anode and the current density is calculated using the area of the sample under test. The current density vs. electric field relationship is collected as the anode voltage is swept across the voltage range and displayed in a corresponding graph. The Fowler-Nordheim plot is derived from the Fowler-Nordheim model for field emission from a flat metal plate in a vacuum and provides evidence the recorded current density is the result of field emission as opposed to thermionic emission.

All the CNT characterization and field emission data is collected and relationships between the quality of the CNT carpet growth and the resulting field emission properties are defined. Due to the results of another research effort that indicate a carbon buffer layer may facilitate CNT growth, the effects of an additional carbon buffer layer between the barrier and the catalyst on CNT quality and field emission is also reported. Finally, the barrier/catalyst combination that makes the best field emission source is determined.

\section{Synthesis of carbon nanotubes}

Since Iijima recognized carbon nanotubes as a by-product of the arc discharge synthesis of fullerenes, the method has been refined for the fabrication of carbon nanotubes. There are three main carbon nanotube fabrication categories that have since been developed:

- Arc discharge synthesis

- Laser ablation synthesis

- Thermal synthesis.

Efforts to produce higher quality, larger quantities, and improve control have yielded a myriad of fabrication methods within each of these categories. The basic physics involved in each fabrication method defines the category. 


\subsection{Arc discharge}

Arc discharge synthesis was the first synthesis method used to reliably produce both MWCNTs and SWCNTs (Bhushan, 2007). Arc discharge or electric arc discharge synthesis is accomplished by generating plasma between two graphite electrodes using a low voltage, high current power supply. The process occurs in an inert atmosphere of He or Ar with pressures ranging from 100 to 1000 torr (O'Connell, 2006). The plasma contains vaporized carbon from the electrodes which then forms carbon nanotubes as it is deposited on the cathode and other areas of the reactor. SWCNTs can only be produced with the addition of a metal catalyst, usually added to the anode via a small hole where a catalyst/graphite powder mixture is placed. CNTs produced using the arc discharge synthesis method require purification before use due to carbon soot and fullerene by-products.

\subsection{Laser ablation}

Laser ablation or laser vaporization, as the name implies, uses a continuous-wave or pulsedwave laser to vaporize a graphite or catalyst metal infused composite graphite target. The process occurs in a quartz furnace at $1200^{\circ} \mathrm{C}$ with a constant flow of inert gas, He or Ar (Tanaka et al, 1999). Vaporized graphite creates a plume with nanoparticles of the metal catalyst which facilitate the growth of SWCNTs. The plume containing the carbon nanotubes and various by-products is swept through the furnace by the inert gas and collected via condensation on a cooled copper $(\mathrm{Cu})$ collector. Again, the presence of carbon by-products makes purification necessary.

\subsection{Thermal synthesis}

Thermal synthesis is a broad category of synthesis methods that rely on thermal energy to produce CNTs. Included in this category is plasma enhanced chemical vapor deposition (PE-CVD), which is a hybrid of plasma based and thermal based synthesis. Due to the wide variety of options and precise control offered by thermal synthesis, chiefly chemical vapor deposition methods; this category has received the most attention from researchers and has yielded the most promising results for controlled CNT fabrication.

CVD in its essence is passing a volatile precursor across a heated substrate to facilitate the deposition of thin films. CVD as a synthesis method could be considered a broad category by itself due to the vast number of variations of this simple process that are available. Two CVD methods, thermal CVD and microwave plasma enhanced CVD, are described below along with the detailed recipes used for CNT synthesis.

All CVD methods require a metal catalyst nanoparticle to facilitate the growth of CNTs. It has been shown that the diameter of the resulting CNTs corresponds to the diameter of the catalyst nanoparticle (Wong et al, 2005). The transition metals $\mathrm{Fe}, \mathrm{Ni}, \mathrm{Co}$, and alloys composed of one or more of these transition metals are the primary catalyst materials. There are a number of methods for depositing the catalyst on the substrate many of which can be easily patterned making it possible to grow CNT arrays at desired locations. Common methods include soaking or spin coating the substrate with $\mathrm{Fe}$, $\mathrm{Co}$, or Mo nitrate oracetate salt and then drying the sample. Sputtering or evaporating a thin layer of catalyst material on the substrate and then subsequently breaking up the catalyst into nanoparticles using a pretreatment consisting either of a high temperature anneal or exposure to a $\mathrm{H}_{2}$ plasma followed by CNT growth by introducing the carbon feedstock gas. This method has been used extensively and a correlation between the deposited layer thickness and resulting 
CNT diameters, thicker layers result in larger diameters, has been documented (Garg et al, 2008; Terranova et al, 2006). The pretreatment time and power, however, must be determined for any given thickness. Contrary to the relationship between catalyst layer thickness and CNT diameter a longer pretreatment time does not always result in smaller particle sizes. Recently, Amana et al. reported that due to Ostwald ripening, pretreatment for too long will result in a lower CNT yield (Amama et al, 2009). Ostwald ripening is a phenomenon related to strain energy that results in small particles shrinking and disappearing over time while large particles increase in size. The Ostwald ripening effect was also documented in a study of MPE-CVD pretreatment of Ni catalyst where an increase in the $\mathrm{H}_{2}$ plasma pretreatment from 5 to 7 minutes resulted in an increase in nanoparticle size from $25 \mathrm{~nm}$ to $40 \mathrm{~nm}$ (Crossley et al, 2010).

\subsubsection{Thermal chemical vapor deposition}

Thermal CVD is the simplest method of chemical vapor deposition. A T-CVD system, as shown in Fig. 1, consists of a furnace, feedstock gasses, and a vacuum pump. Resistive heaters or furnaces operate at temperatures ranging from $500^{\circ} \mathrm{C}$ to $1200^{\circ} \mathrm{C}$ in $\mathrm{T}-\mathrm{CVD}$ systems. A substrate or target with $\mathrm{Fe}, \mathrm{Ni}, \mathrm{Co}$, or an alloy of these three transition metals on the surface is placed in the furnace. An anneal in a non-volatile gas can be performed to form the necessary nanoparticles from thin films of the above mentioned catalyst materials. A carbon feedstock gas such as methane $\left(\mathrm{CH}_{4}\right)$ or carbon monoxide (CO) for SWCNT growth or acetylene $\left(\mathrm{C}_{2} \mathrm{H}_{2}\right)$, ethylene $\left(\mathrm{C}_{2} \mathrm{H}_{4}\right)$, or benzene $\left(\mathrm{C}_{6} \mathrm{H}_{6}\right)$ for MWCNT is flowed across the target substrate (Geckelar \& Rosenburg, 2006). The gas reacts with the catalytic nanoparticles to produce carbon nanotubes.

CVD synthesis occurs at relatively low temperatures that allow conventional substrates to be used, opening up opportunities for integration of CNTs with conventional electronics, optoelectronics, and other applications. With the proper growth conditions, pure CNTs can be produced with yield rates as high as $99 \%$ (O'Connell, 2006). Thus no purification or post processing is necessary unless the catalyst metal must be removed.

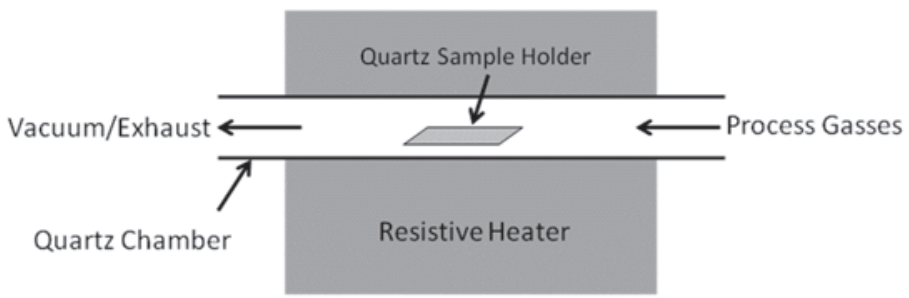

Fig. 1. Thermal chemical vapor deposition system.

\subsubsection{T-CVD process}

The quartz chamber and samples are prepared for growth by evacuating the chamber down to less than 10 mTorr for 15 minutes; after which an $\mathrm{Ar}+\mathrm{H}_{2}$ mix $(95 \%+5 \%)$ is flowed through the chamber to remove unwanted contaminates. The chamber is then evacuated again for an additional 15 minutes. Now that the samples and chamber are prepared, the $\mathrm{Ar}+\mathrm{H}_{2}$ gas mix is flowed while the chamber pressure is stabilized at approximately 90 Torr for the remainder of the entire process. The sample is anealed at $750^{\circ} \mathrm{C}$ to form iron nano- 
islands necessary for CNT synthesis. Next, the growth phase begins with the introduction of the carbon feedstock, in this case an $\mathrm{Ar}+\mathrm{C}_{2} \mathrm{H}_{2}(90 \%+10 \%) \mathrm{mix}$, into the chamber. Growth times ranged from 2 - 20 minutes, resulting in MWCNTs measuring from $5-30 \mu \mathrm{m}$ in length. The growth phase is followed by a short clean phase with $\mathrm{Ar}+\mathrm{H}_{2}$ flow while the chamber temperature is maintained at $750^{\circ} \mathrm{C}$. This removes any weakly bonded residual surface carbon contamination from the sample. Finally, the chamber is cooled to room temperature over a 45-60 minute period with a constant flow of the $\mathrm{Ar}+\mathrm{H}_{2}$ mixture.

\subsubsection{Plasma enhanced chemical vapor deposition}

Plasma Enhanced Chemical Vapor Deposition uses energy from $\mathrm{H}_{2}$ plasma generated from a DC, radio frequency (RF), or microwave power supply, to break down the carbon feedstock gas and facilitate CNT growth at lower temperatures and pressures than simple thermal CVD. Vertically aligned MWCNTs grown on a silicon substrate at temperatures below $330^{\circ} \mathrm{C}$ by microwave plasma enhanced CVD have been documented (Terranova et al, 2006). Typical substrate temperatures range from $400^{\circ} \mathrm{C}$ to $900^{\circ} \mathrm{C}$. PE-CVD systems are capable of growing both SWCNTs and MWCNTs; more importantly these CNTs can be patterned, vertically aligned, and grown on a variety of substrates.

A MPE-CVD system is shown in Fig. 2. Microwave power, up to $1 \mathrm{~kW}$, is transmitted from the generator through a wave guide to a microwave coupler that emits the radiation via the antenna into the CVD chamber. If conditions are correct, the resulting plasma will be centered over the substrate and allow for CNT growth over large areas, $>10 \mathrm{~cm}^{2}$. The CNT growth and plasma formation are dependent upon a number of controllable variables: the microwave power, chamber pressure, gas composition, gas flow, and substrate temperature. The ability to precisely control so many of the growth parameters is the key reason that PECVD has become the most researched synthesis method.

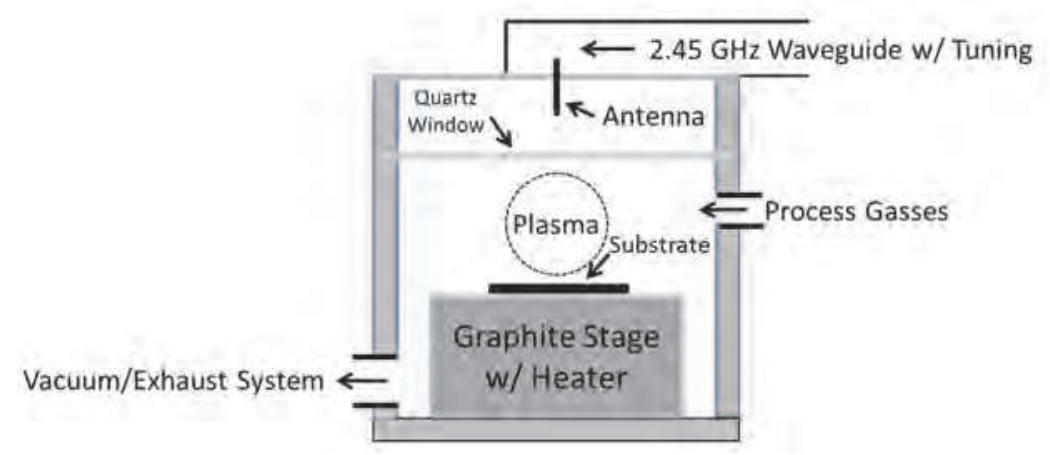

Fig. 2. Microwave plasma enhanced chemical vapor deposition system.

The pretreatment and growth process, as indicated above, are also dependent upon the gas composition, flow rate, and pressure. PE-CVD systems use relatively low pressures ranging from $10-300$ Torr. Lower pressures reduce the presence of residual gasses allowing for more process control. Flow rates depend upon the chamber pressure and gas composition, but recipes reported in the literature range between $100 \mathrm{sccm}$ and $400 \mathrm{sccm}$ total gas flow rate. The gas flow rate and location of the gas inlet can affect the plasma location within the chamber, so an effective flow rate tends to be system specific. 
Gas composition can also vary greatly from process to process. Typical pretreatment recipes consist of pure $\mathrm{H}_{2}$, or some combination of $\mathrm{H}_{2}$ and Ar. However, recent studies have indicated trace amounts, $<1 \%$, of water $\left(\mathrm{H}_{2} \mathrm{O}\right)$ or nitrogen $\left(\mathrm{N}_{2}\right)$ can significantly improve pretreatment effects resulting in greater density and smaller diameter catalyst particles (Amama et al, 2009; Chang et al, 2008). During growth, the gas composition can be even more complex consisting of a carbon feedstock such as, but not limited to, $\mathrm{CO}, \mathrm{CH}_{4}, \mathrm{or}_{2} \mathrm{H}_{4}$, diluted in $\mathrm{H}_{2}, \mathrm{Ar}, \mathrm{N}_{2}, \mathrm{He}$, or some combination. Many different working compositions are possible, but the carbon feedstock gas should be greater than $6 \%$ of the gas mixture to provide enough carbon stock for CNT growth (Nessim et al, 2008). Gas compositions usually contain between $10 \%$ and $40 \%$ feedstock gas. Amorphous carbon, a common unwanted by-product, is more reactive than CNTs which has led to the use of trace amounts of oxidizers such as oxygen $\left(\mathrm{O}_{2}\right), \mathrm{H}_{2} \mathrm{O}$, and hydroxide $(\mathrm{OH})$ and the use of ethanol $\left(\mathrm{C}_{2} \mathrm{H}_{5} \mathrm{OH}\right)$ as a feedstock gas with very promising results $\left(\mathrm{O}^{\prime}\right.$ Connell, 2006).

\subsubsection{MPE CVD process}

The first step in the MWCNT growth process employed for the MPE-CVD system is removing as many potential contaminants as possible. After a substrate is loaded, the chamber is evacuated below 10 mTorr. Then the graphite stage and substrate are heated to $400^{\circ} \mathrm{C}$ in a hydrogen atmosphere of approximately 15 Torr. Once the system has reached a steady state, a catalyst pretreatment phase breaks up the catalyst layer into the nano-islands necessary for $\mathrm{CNT}$ growth. Pretreatment phase exposes the catalyst layer to a $\mathrm{H}_{2}$ plasma induced by $400 \mathrm{~W}$ of microwave power for 5 minutes. The microwave power is terminated at the end of the pretreatment phase and the temperature increased to $650^{\circ} \mathrm{C}$ while maintaining the same gas flow and pressure. The growth phase occurs for 2 minutes at $650^{\circ} \mathrm{C}$, with a hydrogen and methane $\left(\mathrm{CH}_{4}\right) \mathrm{mix}$, and $1000 \mathrm{~W}$ of microwave power. A 2 minute growth results in MWCNTs approximately $2 \mu \mathrm{m}$ in length. The hydrogen flow and chamber pressure are maintained to facilitate cooling back to room temperature.

\subsection{Carbon nanotube growth mechanisms}

Carbon nanotube growth occurs in one of two places and via one of two methods at each location. The type of growth, gas phase or substrate, depends upon the location of the catalyst particle. When the catalyst and CNT formation occur in the chamber atmosphere, it is gas phase growth. Substrate growth is more common and the type of growth applied in this study. It occurs when the catalyst is deposited on the substrate. Both types of CNT growth rely on either surface carbon diffusion or bulk carbon diffusion as the actual growth mechanism.

Surface carbon diffusion is thought to be the growth mechanism for low temperature synthesis methods. The metal catalyst remains solid throughout the growth process and the "cracked" carbon from the feedstock gas diffuses around the particle surface. The carbon nanotube nucleates around the side of the metal particle. Since there is an abundant source of carbon from the feedstock gas, carbon continues to break down on the catalyst, diffuse around the particle, and facilitate nanotube growth ( $\mathrm{O}^{\prime}$ Connell, 2006).

In bulk carbon diffusion the catalyst can be either a solid or liquid nano-droplet depending on the transition metal and process temperature. The "cracked" carbon feedstock gas is dissolved by the catalyst until saturation at which point a carbon nanotube forms on the outer surface. This is a vapor - liquid - solid transition, where the feed stock gas is broken down into a hydrocarbon vapor, then forms a metal-carbon liquid when dissolved by the catalyst, and finally transitions to a crystalline carbon solid in the form of a nanotube. 
Substrate growth through surface carbon diffusion is the most likely growth type and mechanism for CVD methods. This stems from the goals of most CVD growth methods which is to keep the temperature low to facilitate more substrate options and the use of deposited catalysts for controlled growth. Regardless of the growth mechanism substrate growth can occur at either end of the nanotube. If the stiction of the catalyst particle to the substrate is sufficiently large the particle will stay adhered to the substrate and the CNT will form by base growth. Tip growth occurs when the catalyst particle has insufficient stiction. In this case, the CNT adheres to the substrate and the catalyst forms the tip of the growing CNT. Fig. 3 illustrates both substrate growth conditions. While both base and tip growth can be observed with SWCNT and MWCNT fabrication, tip growth is dominant in MWCNTs and base growth is dominant in SWCNTs. This is largely due to the forces exercised on the catalyst nanoparticle at the time of growth; SWCNTs cannot overcome the stiction of the catalyst while typically MWCNTs can. Base growth in MWCNTs is often observed if the catalyst nanoparticles are patterned or formed as a function of deposition as these particles have a significantly higher stiction than nanoparticles formed by a granulation process. Tip growth is expected for all cases in this effort due to the catalyst pretreatment methods used to create the nanoparticle nucleation sites.

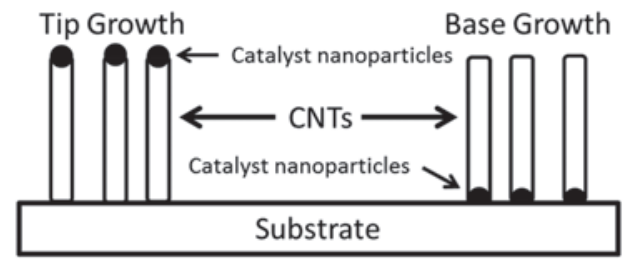

Fig. 3. Carbon nanotube tip and base growth from a substrate.

In theory, CNT growth should be capable of continuous growth as long as the feedstock carbon gas is supplied. In reality, there are a number of conditions that can occur and impede or prematurely terminate the growth process. Growth can only occur at the catalyst; base growth can be slowed and even stopped due to slow or loss of diffusion of carbon down to the nanoparticle. The carbon feedstock gas can supply an over abundance of carbon causing the deposition of amorphous carbon which then coats the catalyst and subsequently impedes and ultimately stops CNT growth. Occasionally, the catalyst can form graphitic shells instead of a carbon nanotube which will block access to more carbon ending the growth process. These are causes of poor growth conditions and can usually be avoided by appropriately adjusting the growth parameters.

\section{Carbon nanotube field emission}

CNTs have many unique properties ideal for field emission such as narrow diameters, high aspect ratios, high temperature stability, good conductivity, and structural strength. Carbon nanotubes make excellent electron emitters, not because of a low work function, but due to the extremely high local electric field that forms at the small diameter tips. Single walled CNTs have diameters from $0.6 \mathrm{~nm}$ to $1.4 \mathrm{~nm}$ and multi-walled CNTs can range from $12 \mathrm{~nm}$ to more than $100 \mathrm{~nm}$ in diameter. Both types of CNTs can range from tens of nanometers to microns and even millimeters in length resulting in incredible aspect ratios. The resulting 
focus of the electric field at the tips of the CNTs is often referred to as the electric field enhancement factor. Previous research as summarized by Bonard et al has shown multiwalled CNTs to be more robust emitters than single walled CNTs (Bonard et al, 2001). Thus, only multi-walled CNTs are grown in this field emission effort. While the conductivity of single walled CNTs can be metallic or semiconducting depending on the nanotube structure, all multi-walled CNTs are considered metallic conductors because some of the nested nanotubes making up the multi-wall CNT will be metallic. This makes attempts to control or consider the chirality or type of CNTs grown unneccessary; another benefit of using MWCNTs for field emission instead of SWCNTs.

Potential commercial applications are the driving forces behind CNT field emission research. Different applications lead to different requirements, however, there are a few figures of merit that can be used to assess the relative quality of a CNT emitter. Current density is the value most often given when reporting results of CNT field emission research. Current density without knowing the associated electric field is of little use. The area used to calculate current density is also important. The current density from a single CNT can be remarkably high, basing the potential emission from an array of nanotubes on a single CNT has led to current density predictions as unfathomably high as $10^{7} \mathrm{~A} / \mathrm{cm}^{2}$ (Zhu et al, 1999). But, due to screening effects, getting every CNT in a large area to emit electrons has proven to be a much more difficult task making current density measuremtents of individual CNTs useless in predicting the field emission from large areas of CNTs.

Other important parameters that aid in comparing results are the turn-on field, $\mathrm{E}_{\mathrm{to}}$; the electric field enhancement factor, $\beta$; and the total current density at a high electric field strength. The turn on field, much like diodes and transistors, is the field required to achieve a given current density. A current density of $1 \mu \mathrm{A} / \mathrm{cm}^{2}$ is used to determine $\mathrm{E}_{\mathrm{to}}$. The enhancement factor is a measure of the increase in the localized electric field at the CNT emission site compared to the macro electric field of the system. An explanation and the method of calculating $\beta$ from measured data is detailed below in Section 3.2.

CNT field emission is achieved with a diode configuration, shown in Fig. 4, where the CNTs act as the cathode emitters and a copper collector plate serves as the anode. The diode configuration is often used for its simplicity and to characterize the field emission properties of unpatterned CNT carpets. Field emission using this configuration often requires high voltages to establish the necessary electric fields, due to the relatively large distance, $d$, between cathode and anode.

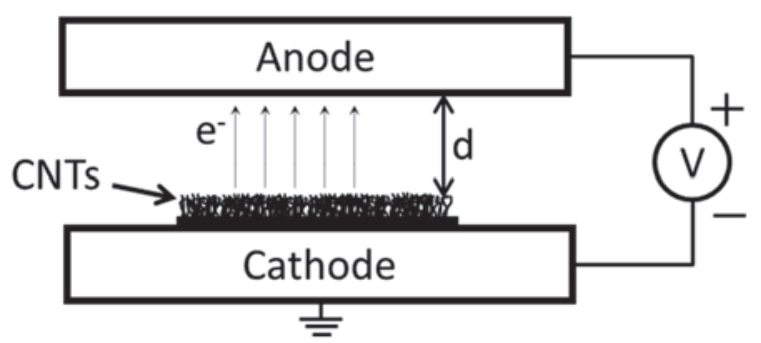

Fig. 4. Diode configured field emission schematic.

In his seminal publication, de Heer estimated a mere $0.1 \%$ of the total CNTs were emitting (de Heer et al, 1995). They concluded only a tiny fraction of CNTs was sharp enough to be 
efficient emitters. Since then, the phenomenon has been attributed to electrostatic screening effects (Leonard, 2008). As the number of CNTs in the emission area increases the space between them decreases limiting the electric field penetration around the CNTs. This limited field penetration, in turn, reduces the localized electric field enhancment significantly. 2D electrostatic simulations of field penetration between CNTs, visualized in Fig. 5, resulted in a $60 \%$ reduction in the localized electric field of nanotubes separated by $50 \mathrm{~nm}$ compared to a single CNT (Crossley et al, 2009). A uniform dense growth of CNTs effectively becomes the equivalent of a flat surface negating many of the advantages carbon nanotubes offer as a field emission source. Many optimized separation distances have been proposed for ideal CNT field emission ranging from a separation equal to the CNT height to a separation 5 times the CNT height, however it is widely accepted that some separation is necessary to reduce screening effects for efficient field emission (Nilsson et al, 2000; Smith \& Silva, 2009; Suh et al, 2002). When unpatterned CNT carpets are grown, as is the case for this current study, the process of granulating the catalyst layer can be adjusted to affect the resulting nano-particles size and areal density. Both of which can have significant effects on field emission properties.

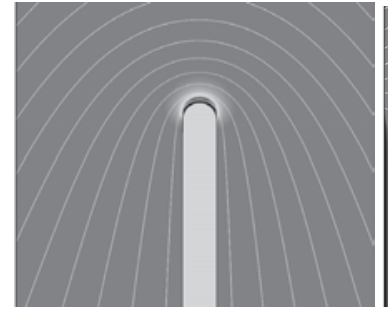

(a)

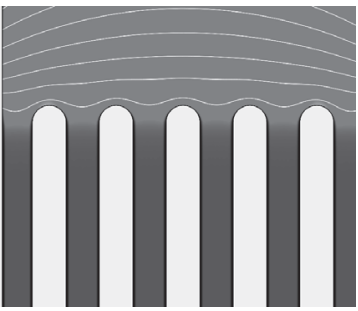

(b)

Fig. 5. Electrostatic field penetration for (a) Single CNT and (b) CNTs with $50 \mathrm{~nm}$ spacing.

\subsection{Fowler-Nordheim plot and field enhancement}

The Fowler-Nordheim (FN) model describes the current or current density obtained from electrons tunneling through a metal-vacuum potential barrier at $0 \mathrm{~K}$. Other conditions for the Fowler-Nordheim model include tunneling from a flat metal surface; the metal or emitter is modeled as a free electron gas; and the potential barrier height is independent of the applied voltage (Leonard, 2008). Although the actual potential barrier is a combination of the image force potential and the external force potential; a simplifying triangular barrier, shown in Fig. 6, is adequate for the approximations used to derive the Fowler-Nordheim plot.

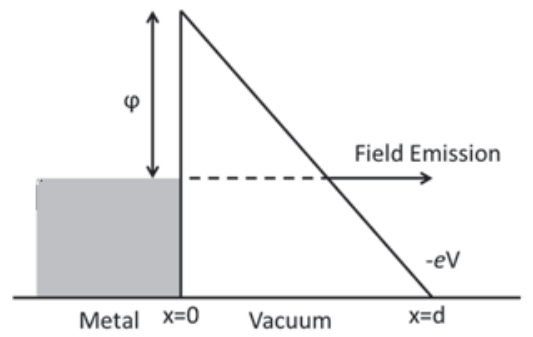

Fig. 6. Fowler-Nordheim triangular potential barrier approximation. 
The simplified FN equation for current density from field emission is shown below.

$$
J=\frac{a F^{2}}{t^{2}(y) \varphi} e^{\left(\frac{-b \varphi^{3 / 2}}{F} v(y)\right)}
$$

Where current density, $J$, is in $\mathrm{A} / \mathrm{cm}^{2}, a$ and $b$ are constants of $1.54 \times 10^{-6} \mathrm{AV}^{-2} \mathrm{eV}$ and $6.83 \times 10^{7} \mathrm{eV}^{-3 / 2} \mathrm{Vcm}^{-1}, t(y)$ and $v(y)$ are tabulated functions, $F$ is the applied electric field, and $\varphi$ is the work function. The function $t(y)$ is close to and so often set equal to unity. The Nordheim function, $v(y)$, varies significantly; however, in what is considered an improper or gross simplification $v(y)$ is also commonly set to unity (Paulini et al, 1993).

The applied field is adjusted to account for the sharp tip of carbon nanotubes by including a field enhancement factor $\beta$. The field enhancement factor takes into account the microscopic or localized field at the emitter tip. Electric field lines concentrate at the area of small radius of curvature resulting in much higher localized field potentials. The applied field $F$, is considered a uniform field such as between two parallel plates and is defined defined as:

$$
F=\frac{V}{d}
$$

where $\mathrm{V}$ is the applied voltage and $\mathrm{d}$ is the separation distance as shown in Fig. 6. The inclusion of the enhancement factor gives the localized field shown in Equation (3).

$$
F_{\text {Local }}=\frac{\beta V}{d}
$$

Field enhancement for field emitting CNTs has a wide range of reported values from 1000 to 8000 and even as high as 26000 (Chhoker et al, 2007; Seelaboyina et al, 2008). Issues with determining field enhancement will be discussed below.

Including the simplifications mentioned above, the field enhancement factor, and converting from current density to current by including the total emission area the following FN current relationship is obtained:

$$
I \approx \frac{a S \beta V^{2}}{d^{2} \varphi} e^{\left(\frac{-b d \varphi^{3 / 2}}{\beta V}\right)}
$$

where $S$ is the emitting surface area in $\mathrm{cm}^{2}$. By dividing by $V^{2}$ and taking the natural $\log$ Equation (4) takes the form:

$$
\ln \left(\frac{I}{V^{2}}\right) \approx-\frac{b d \varphi^{\frac{3}{2}}}{\beta V}+\ln \left(\frac{S a \beta^{2}}{d^{2} \varphi}\right)
$$

Equation (5) is easily recognized as the equation of a line for $\ln \left(I / V^{2}\right)$ as a function of $1 / V$. Thus, FN field emission can be shown if a line results from plotting measurements of emission current as a function of the applied voltage in the form $\ln \left(I / V^{2}\right)$ versus $1 / V$. This is often referred to as a Fowler-Nordheim plot--see Fig. 7 as an example--and is used to demonstrate field emission as opposed to thermionic emission. 


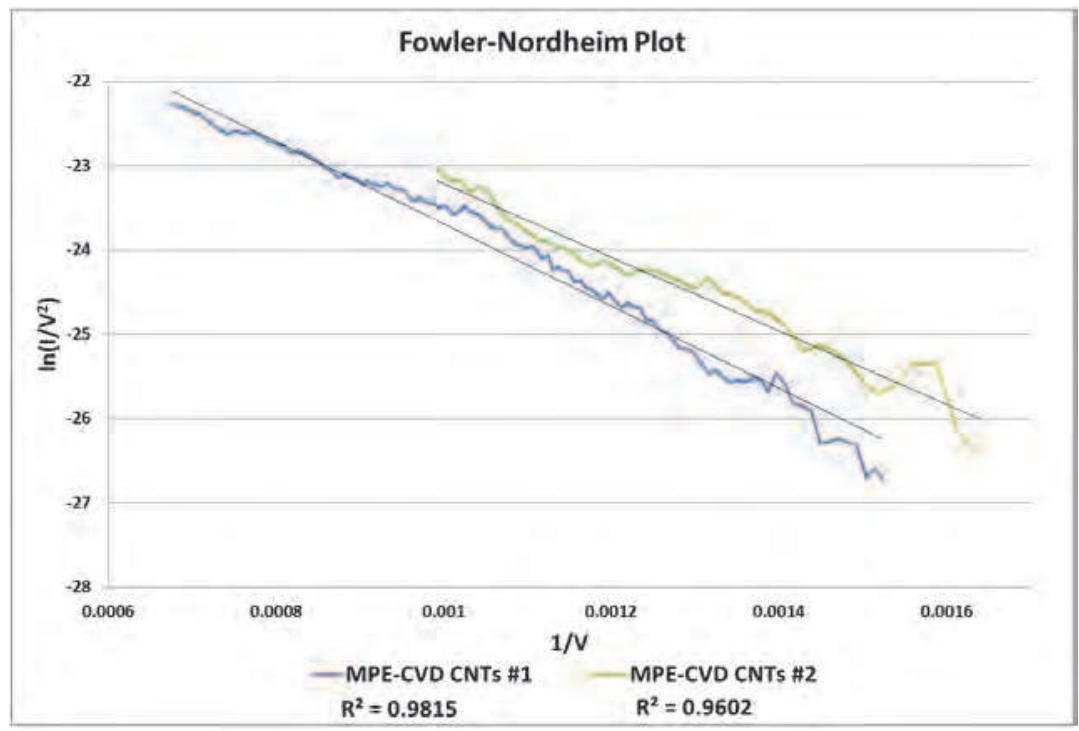

Fig. 7. Fowler-Nordheim Plot of MPE-CVD grown CNT samples with linear trendline.

The slope of the FN plot, given in Equation (6), can be used to determine the field enhancement factor, $\beta$. There is one caveat to this method of calculating the enhancement factor: the work function of the CNTs must also be known. There have been several methods employed to measure the work function of CNTs. Ultraviolet photoemission spectroscopy was used to measure the work function of SWCNT bundles at $4.8 \mathrm{eV}$, while other reported values have ranged from $4.3 \mathrm{eV}$ to $5.7 \mathrm{eV}$ (Suzuki et al, 2000). Xu et al used a transmission electron microscope with a unique tungsten needle sample holder to measure the work function of individual MWCNTs grown via MPE-CVD. The measurements indicated differences in the physical structure at the tube tips caused variations in the work functions from $4.51 \mathrm{eV}$ to $4.78 \mathrm{eV}$ (Xu et al, 2005). Only MWCNTs are considered in this study as well, so a work function of $4.65 \mathrm{eV}$ is used in all calculations. Thus, for MWCNT samples \#1 and \#2 shown in Fig. 7 the resulting field enhancement factors are 2390 and 3460 respectively. A higher $\beta$ is indicative of more efficient field emission. The increased localized electric field results in field emission at lower applied voltages.

$$
m=\frac{b d \varphi^{\frac{3}{2}}}{\beta}
$$

With all the assumptions and simplifications listed, it is obvious the FN model is not accurate for field emission from CNT tips. However, many experimental and theoretical efforts to develop an accurate model have yet to produce a conclusive result; while the FN relationship given above has been proven experimentally to be a good fit for I-V characteristics of CNT emitters of all kinds (Leonard, 2008). The sheer number of methods and processes used to achieve emission and the often seemingly contradictory results of different research efforts have made a functional model for electron emission from carbon nanotubes a difficult and elusive task. 


\section{Substrate preparation}

A low resistivity Si substrate is prepared by first depositing a barrier layer and then the catalyst material. A low resistivity barrier layer provides an adhesion layer, electric contact, and diffusion barrier preventing the formation of silicides. Previous research has indicated that the barrier layer material can significantly affect the granulation process (Crossley et al, 2010). Thus, the barrier material and thickness can have significant effects on CNT growth. Studies show that barriers such as silicon dioxide and aluminum oxide produce high yields of CNTs, but due to their insulating nature they were not considered for use as barrier layers in this study. Instead conductive barriers of titanium and chromium that meet all the above barrier layer requirements were used. One set of wafers had a $20 \mathrm{~nm}$ layer of sputtered amorphous carbon deposited on the barrier layers to determine if the carbon layer would promote more uniform CNT growth and limit possible adverse effects caused by different thicknesses and types of barrier materials. Silicon wafers were sputtered with Ti or $\mathrm{Cr}$ barriers of $25 \mathrm{~nm}$ and $100 \mathrm{~nm}$ followed by iron catalyst layers of $2 \mathrm{~nm}$ and $10 \mathrm{~nm}$. The $100 \mathrm{~nm}$ thick barriers were used to provide better electrical conduction for field emission and further micro-fabrication processes planned for future research. Two catalyst thicknesses were used because there is a strong correlation between the thickness of the catalyst layer, the size of the nano-particles after granulation, and the diameter of the resulting CNTs (Crossley et al, 2010; Wong et al, 2005). All wafers prepared as described above were used in the T-CVD process. CNTs grown by MPE-CVD used a $20 \mathrm{~nm}$ titanium barrier with a $10 \mathrm{~nm}$ nickel catalyst layer determined through a previous research effort (Crossley et al, 2010).

\section{Results}

Identical sets of samples cleaved from wafers prepared as described in Section 4 were processed in two separate manners. The first set of samples was only processed through the granulation of the catalyst, the anneal for T-CVD and the hydrogen plasma pretreatment for MPE-CVD described in Sections 2.3.2 and 2.3.4 respectively. The samples were cooled, removed, and the resulting nano-islands imaged by SEM. Due to the anneal temperature and required cooling time the nano-islands imaged will not be identical to the samples which proceed directly from anneal to CNT growth. However, analysing the size and areal density of the catalyst nano-islands provides a good indication of the resulting diameter and density of CNTs.

The second set of samples was processed through both granulation and growth phases of each CVD method. The resulting multi-walled CNT carpets were characterized by Raman spectroscopy and imaged by SEM to determine their quality. After this characterization of CNT quality, the field emission properties of the CNT carpets were measured in a custom built field emission test chamber. Correlating the field emission properties with CNT characterization provided insight into what properties of the CNT carpets are key to making the most efficient and effective field emission devices.

\subsection{CNT characterization}

Raman spectroscopy is the first step in characterizing the CNT carpet samples. Raman spectroscopy is a light scattering technique where an excitation laser is focused on the material of interest and the interaction of the light photon with the material results in a 
small amount of light scattering at a slightly different frequency. This slight shift in frequency is called a Raman shift and provides a unique finger print of the material. Raman spectroscopy has been used for decades to identify carbon materials; and more recently has been shown capable of providing a myriad of information about carbon nanotubes such as doping, defects, diameter, chirality, and curvature (Dresselhaus et al, 2010).

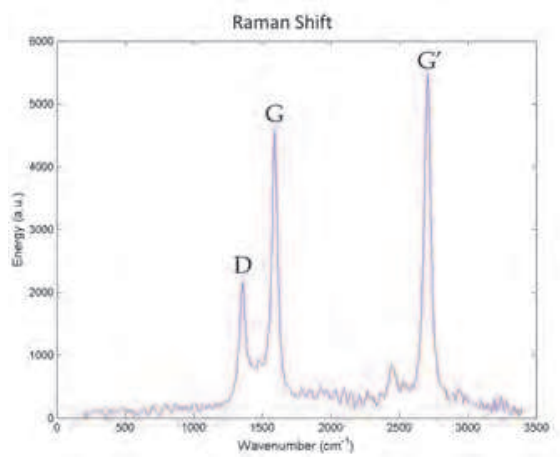

(a)

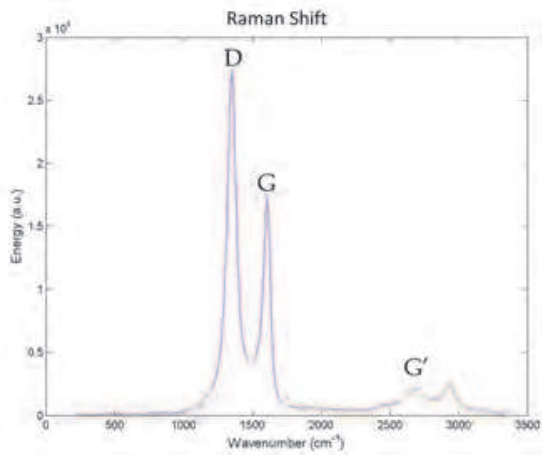

(b)

Fig. 8. Raman shift with D, G, and G' peaks labeled; (a) good quality or low defect MWCNTs grown by T-CVD and (b) poor quality or defect prone MWCNTs grown by MPE-CVD.

Raman spectroscopy was performed using a $532 \mathrm{~nm}$ green excitation laser. CNTs have a Raman shift with three primary peaks of interest, D-band, G-band, and G'-band, that can be used to determine the CNT carpet quality (DiLeo et al, 2007). The G-band peak is located at approximately $1580 \mathrm{~cm}^{-1}$ and is a result of ordered C--C bonds, indicative of defect free graphene and carbon nanotubes. The D-band, approximately $1350 \mathrm{~cm}^{-1}$, is a disorder induced peak caused by defects in the carbon structure. A relative determination of CNT quality can be found by comparing the ratio of the D-band intensity $\left(\mathrm{I}_{\mathrm{D}}\right)$ over the G-band intensity $\left(\mathrm{I}_{\mathrm{G}}\right)$. The $\mathrm{G}^{\prime}$-band, approximately $2700 \mathrm{~cm}^{-1}$, is dispersive but it is the result of a double resonance process and is also very prevalent in Raman shift data for graphene. DiLeo et al propose that in MWCNTs disorder or defects will reduce this double resonance process decreasing the $G^{\prime}$ peak intensity providing a more accurate indication of CNT quality from the ratio of the $G^{\prime}$ peak intensity $\left(I_{G^{\prime}}\right)$ over $I_{D}$ (DiLeo et al, 2007). The ratio, $\mathrm{I}_{\mathrm{G}^{\prime}} / \mathrm{I}_{\mathrm{G}}$, was also recorded to determine any correlation with field emission properties. Raman spectroscopy by itself does not provide a complete picture of carbon nanotube quality. SEM images are also necessary to determine the areal density, presence of amorphous carbon, and physical characteristics of the CNTs.

Fig. 8 (a) shows the Raman shift of T-CVD grown CNTs from $10 \mathrm{~nm}$ of Fe catalyst on a $100 \mathrm{~nm}$ Ti barrier with the $\mathrm{D}, \mathrm{G}$, and $\mathrm{G}^{\prime}$ peaks labelled. The $\mathrm{I}_{\mathrm{D}} / \mathrm{I}_{\mathrm{G}}$ and $\mathrm{I}_{\mathrm{G}^{\prime}} / \mathrm{I}_{\mathrm{D}}$ ratios, 0.47 and 2.52 respectively, represent the best quality MWCNTs of all the different catalyst/barrier combinations considered. Fig. 8 (b) is the Raman shift of MWCNTs grown by MPE-CVD and as is common for CNTs grown in this manner the D peak intensity is much greater than the $G$ peak. The high D peak intensity is from first order dispersion effects caused by defects in the CNTs. These same defects prevent the secondary double resonance dispersion effects that cause the $G^{\prime}$ peak, which is correspondingly low. With $I_{D} / I_{G}$ and $I_{G^{\prime}} / I_{D}$ ratios of 
1.58 and 0.1 , these MWCNTs are the poorest quality of all the different catalyst/barrier combinations considered for both growth methods, T-CVD and MPE-CVD. However, as mentioned above, Raman spectroscopy provides a relative measure of the quality of the CNTs, but SEM imagery is required to complete the characterization of the CNTs.

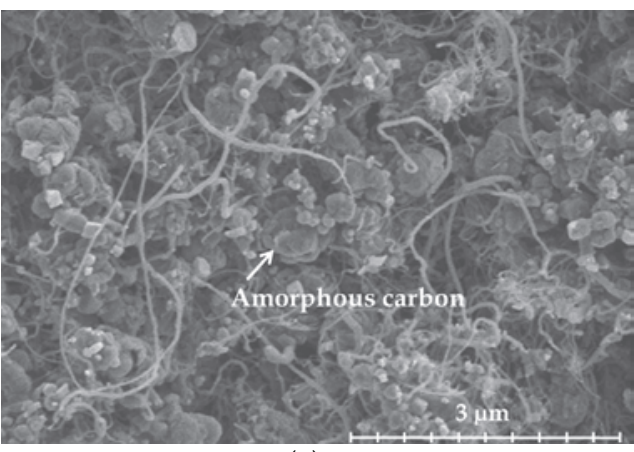

(a)

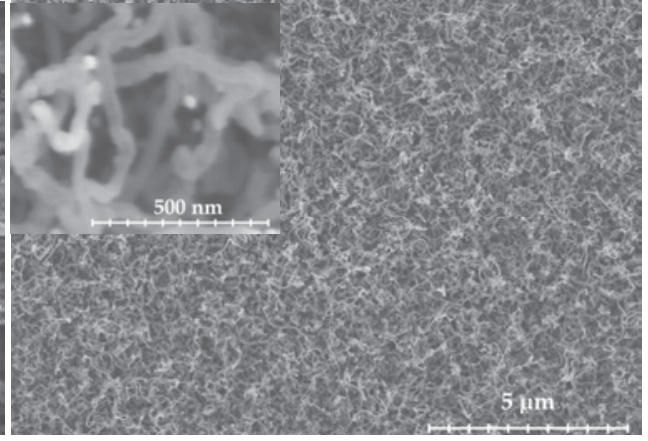

(b)

Fig. 9. SEM micrograph of MWCNT growth by (a) T-CVD from $10 \mathrm{~nm}$ Fe catalyst on a $100 \mathrm{~nm}$ Ti barrier and (b) MPE-CVD from $10 \mathrm{~nm}$ Ni catalyst on a $20 \mathrm{~nm}$ Ti barrier.

SEM micrographs of the above two samples, the highest and lowest quality CNT samples per Raman spectroscopy, are shown in Fig. 9. The T-CVD sample in Fig 9 (a) has sparse CNT growth with large amounts of amorphous carbon deposited on the substrate surface. Also, the diameter of the CNTs varies widely from $\sim 20-100 \mathrm{~nm}$. While the CNTs that are present may have few defects, the overall quality of the CNT carpet is poor due to the low areal density, amorphous carbon deposits, and diverse diameters. The MPE-CVD sample in Fig. 9 (b) is a dense CNT carpet with no visible amorphous carbon deposits and much more uniform CNT diameters at approximately $50 \mathrm{~nm}$ and lengths of $1-2 \mu \mathrm{m}$. The $500 \mathrm{~nm}$ scale inset shows a close up view of the MWCNTs. The bright spots at the CNT ends are caused by catalyst particles that facilitated tip growth and now form a cap or plug at the CNT tip. These CNTs are riddled with growth defects, but the CNT carpet is much better quality than the sparse growth in Fig. 9 (a).

Table 1 below lists the Raman shift intensity ratios and SEM based qualitative assessment of CNT growth for all catalyst/barrier combinations. The chrome barrier of both 25 and 100 $\mathrm{nm}$ thicknesses performed poorly. Two combinations, Cr-100/Fe- $2 \mathrm{~nm}$ and $\mathrm{Cr}-100 / \mathrm{C}-$ 20/Fe-2 nm, did not grow CNTs. In Fig. 10 the SEM micrograph of the catalyst after the anneal and growth process revealed poor granulation with the expected nano-islands spanning hundreds of nanometers instead of the desired $25-50 \mathrm{~nm}$. The Cr-100/Fe- $13 \mathrm{~nm}$ sample grew a sparse CNT carpet with CNT diameters in excess of $100 \mathrm{~nm}$ putting them closer to carbon fibers rather than carbon nanotubes. The addition of $20 \mathrm{~nm}$ of carbon improved the anneal process. The Cr-100/C-20/Fe-10 nm sample showed an improvement over the $\mathrm{Cr}-100 / \mathrm{Fe}-10 \mathrm{~nm}$ sample in both $\mathrm{D} / \mathrm{G}$ and $\mathrm{G}^{\prime} / \mathrm{D}$ intensity ratios and the CNT diameters were reduced to less than $100 \mathrm{~nm}$.

The $25 \mathrm{~nm}$ chrome samples had poor overall CNT carpets. The Cr-25/Fe-10 nm sample was almost identical to the Ti-100/Fe-10 nm sample shown in Fig. 9 (a), sparse growth of low defect CNTs with large deposits of amorphous carbon. The addition of $20 \mathrm{~nm}$ of carbon had little effect, improving the Raman shift intensity ratios slightly in the Cr-25/C-20/Fe-2 nm 
case and making the $D / G$ and $G^{\prime} / D$ intensity ratios slightly worse for the Cr-25/C-20/Fe$10 \mathrm{~nm}$ case. In both cases there was no appreciable difference in the SEM micrographs between the carbon and no carbon buffer layer combinations.

\begin{tabular}{|c|c|c|c|c|c|c|c|c|}
\hline \multicolumn{2}{|c|}{$\begin{array}{c}\text { Barrier Layer Type \& } \\
\text { Thickness (nm) }\end{array}$} & $\begin{array}{c}\text { Catalyst } \\
(\mathrm{nm})\end{array}$ & $\mathrm{D} / \mathrm{G}$ & $\mathrm{G}^{\prime} / \mathrm{G}$ & $\mathrm{G}^{\prime} / \mathrm{D}$ & \multicolumn{2}{|c|}{$\begin{array}{c}\text { Areal } \\
\text { Density }\end{array}$} & $\begin{array}{c}\text { Overall } \\
\text { Growth }\end{array}$ \\
\hline \multirow{4}{*}{ Chrome } & 100 & 13 & 0.69 & 0.82 & 1.20 & Low & Poor \\
\cline { 2 - 8 } & 100 & 2 & \multicolumn{5}{|c|}{ No CNT Growth } \\
\cline { 2 - 8 } & 25 & 10 & 0.48 & 1.11 & 2.31 & Low & Poor \\
\cline { 2 - 8 } & 25 & 2 & 0.88 & 0.72 & 0.82 & Medium & Poor \\
\hline \multirow{3}{*}{$\begin{array}{c}\text { Chrome \& } \\
\text { Carbon }\end{array}$} & $100 \& 20$ & 10 & 0.60 & 1.09 & 1.82 & Medium & Good \\
\cline { 2 - 8 } & $100 \& 20$ & 2 & \multicolumn{6}{|c|}{ No CNT Growth } \\
\cline { 2 - 8 } & $25 \& 20$ & 10 & 0.54 & 1.19 & 2.19 & Low & Poor \\
\hline \multirow{3}{*}{ Titanium } & 100 & 10 & 0.47 & 1.19 & 2.53 & Low & Poor \\
\cline { 2 - 8 } & 100 & 2 & 0.56 & 0.95 & 1.71 & Low & Poor \\
\cline { 2 - 8 } & 25 & 10 & 0.48 & 0.99 & 2.06 & Medium & Good \\
\cline { 2 - 8 } & 25 & 2 & 0.90 & 0.86 & 0.96 & Medium & Good \\
\hline Titanium \& & $25 \& 20$ & 10 & 0.48 & 1.09 & 2.26 & Low & Poor \\
\cline { 2 - 8 } Carbon & $25 \& 20$ & 2 & 1.09 & 0.69 & 0.63 & Medium & Poor \\
\hline MPE-CVD \#1 & Ti 20 & Ni 10 & 1.26 & 0.20 & 0.16 & High & Good \\
\hline MPE-CVD \#2 & Ti 20 & Ni 10 & 1.58 & 0.15 & 0.09 & High & Good \\
\hline
\end{tabular}

Table 1. Raman shift peak intensity ratios and SEM based qualitative assessment of CNT growth for all catalyst/barrier combinations.

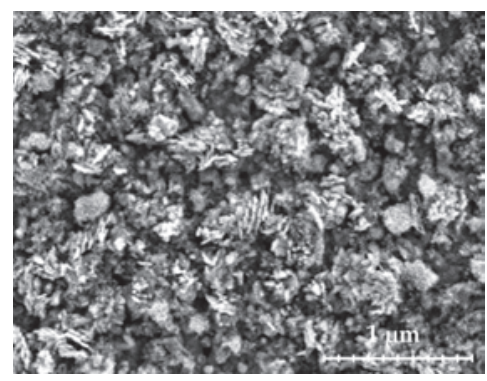

Fig. 10. SEM micrograph of the Cr-100 nm/Fe-20 nm sample after anneal and growth processes.

All titanium barrier combinations produced CNT carpets; although the $100 \mathrm{~nm}$ titanium samples had anneal issues that resulted in low defect CNTs with poor overall CNT carpet growth as discussed above and shown in Fig. 9 (a). The $25 \mathrm{~nm}$ titanium barrier proved most effective for growing MWCNT carpets. The Ti-25/Fe-10 nm sample, shown in Fig. 11, produced a medium to high density, low defect CNT carpet with little to no amorphous carbon and fairly uniform diameters. The Ti-25/Fe- $2 \mathrm{~nm}$ carpet had a lower areal density with more CNT defects as evidenced by the Raman shift ratios. Adding the carbon thin film 
had little effect on the Raman shift characteristics and made the overall CNT carpets less dense with more amorphous carbon deposits; for the Ti-25/C-20/Fe-10 nm case the amount of amorphous carbon was significantly increased.
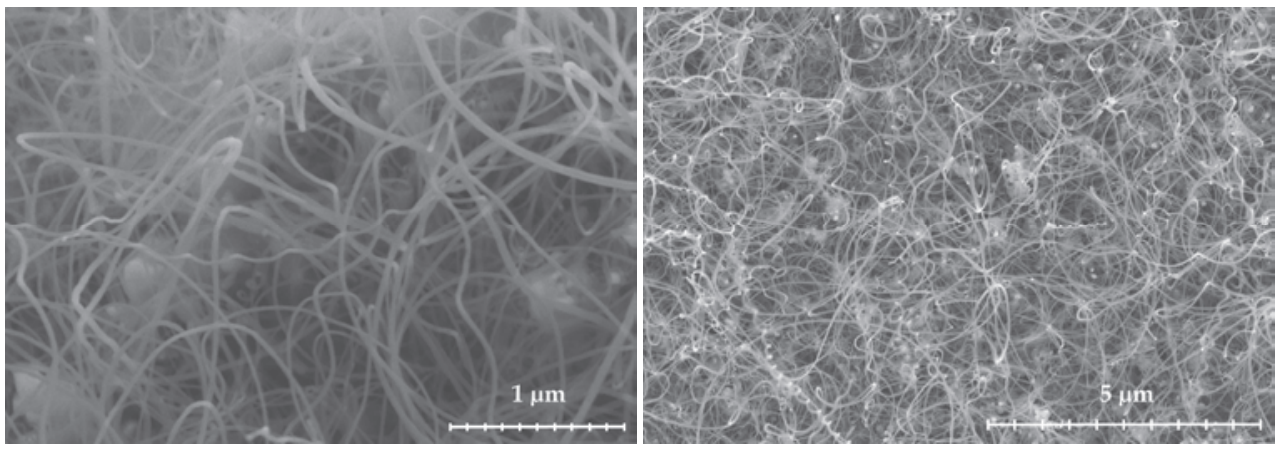

Fig. 11. SEM micrographs of CNT carpet grown by T-CVD on Ti-25/Fe-10 nm sample.

Using thermal CVD, the $25 \mathrm{~nm}$ Ti is clearly the most effective barrier with the Ti-25/Fe$10 \mathrm{~nm}$ sample producing the cleanest, defect free CNT carpets out of all the barrier/catalyst combinations. The $100 \mathrm{~nm} \mathrm{Ti}$ and both $\mathrm{Cr}$ barrier thicknesses have different thermal properties that will require extensive study to determine an appropriate anneal process for each barrier/catalyst combination to achieve catalyst nano-islands conducive to CNT synthesis. The addition of sputtered carbon as a buffer between the barrier and the catalyst did not reduce the effects of changes to the barrier, but rather added another change to the thermal properties during the anneal process. The addition of carbon on the chrome barrier improved the anneal process and subsequent CNT growth. However, the carbon layer degraded the overall quality of the CNT carpet growth when added to the Ti barrier.
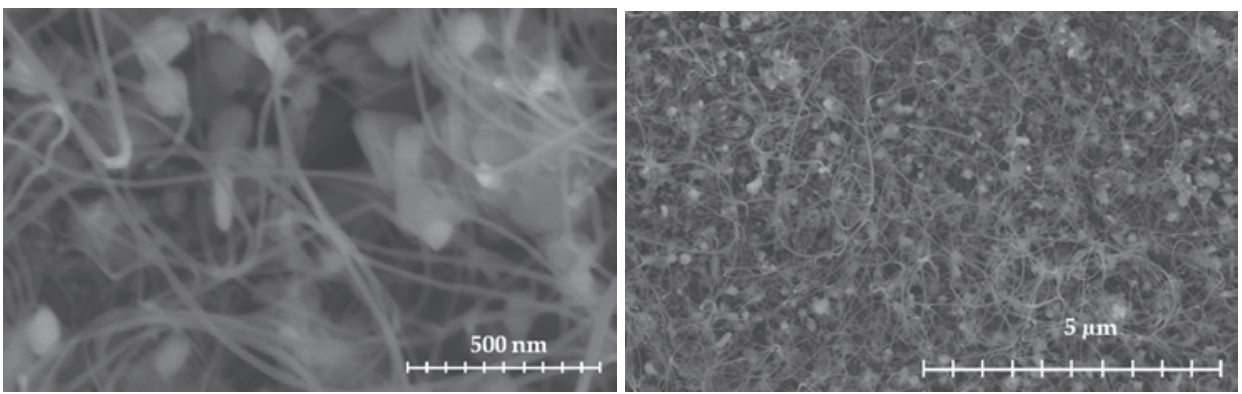

Fig. 12. SEM micrographs of CNTs grown by T-CVD for 2 minutes on Ti-25/Fe-10 nm.

The MPE-CVD grown CNT carpets are defect prone, but have no discernable amorphous carbon and have the highest areal density. MPE-CVD also offers precise control of the CNT growth conditions in the MPE-CVD process making it possible to achieve CNT carpets with uniform lengths. The CNT carpets of the two samples considered are approximately $2 \mu \mathrm{m}$ in length. The short length is necessary for future integration into gated field emission devices. The T-CVD process does not have as much control over the growth conditions of the CNTs. Fig. 12 shows a Ti-25/Fe-10 nm sample with a CNT carpet from a 2 minute 
growth. The CNTs are less dense than the 20 minute growth shown in Fig. 11, but still are in excess of $10 \mu \mathrm{m}$ in length with many exceeding $15 \mu \mathrm{m}$. Integration of CNTs grown by TCVD into gated field emission devices is unlikely until the lack of uniformity and control of the CNT lengths is resolved.

\subsection{Field emission}

After CNT characterization with Raman spectroscopy and SEM imagery, the CNT carpets were tested for field emission in a diode configured test chamber similar to the schematic shown in Fig. 4. The chamber is evacuated to less than $1 \times 10^{-6}$ torr before field emission testing. The total current is measured on both the supply and ground side of the device under test to detect any potential leakage currents. A complete test run consists of increasing the extraction voltage in $10 \mathrm{~V}$ increments, equates to $\sim 0.05 \mathrm{~V} / \mu \mathrm{m}$, from zero up to a preset voltage. At each voltage, after a pause for the system to reach a steady state, 100 measurements are taken at $0.01 \mathrm{~s}$ intervals and averaged before proceeding to the next voltage increment. After reaching the preset voltage, the process is reversed and measurements are taken as the voltage steps back down to zero. The initial preset voltage is $700 \mathrm{~V}$ or $\sim 3 \mathrm{~V} / \mu \mathrm{m}$. However, if at any point during the test damage occurs to the CNTs, determined by visual arching at the CNT carpet or by sudden current spikes, the test is immediately terminated and the highest voltage measured is recorded as the maximum for the device under test. If a device is tested to $700 \mathrm{~V}$ with little or no field emission measured, the set voltage is increased for further testing. The system maximum voltage is $5000 \mathrm{~V}$ or $\sim 23 \mathrm{~V} / \mu \mathrm{m}$. The highest electric field applied for this effort was $5.34 \mathrm{~V} / \mu \mathrm{m}$. Increasing the electric field strength too far damages the CNTs and causes permanent degradation in field emission performance.

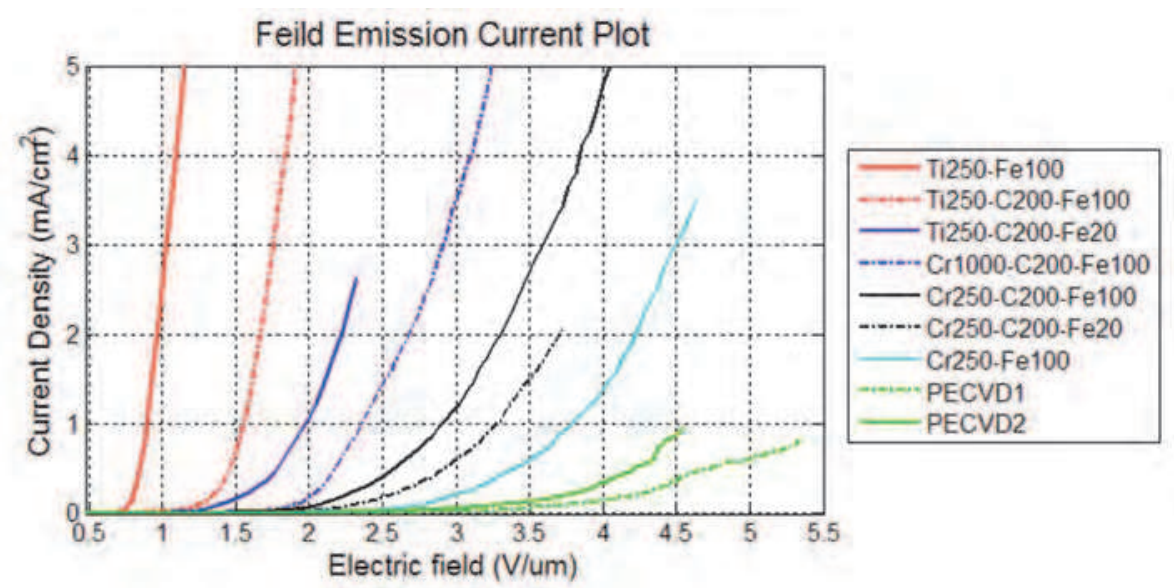

Fig. 13. Field emission current density plot for selected samples from Table 2.

The collected field emission data displayed in Fig. 13 visually identifies the strong field emission performers. The measurements are collected as current and matching voltage. The total field emission area is determined by measuring the sample area under the collector plate during the field emission tests using a set of precision callipers. The electric field is calculated simply by dividing the applied voltage by the distance, $d$, between the device 
under test and the collector plate $(d=215 \mu \mathrm{m})$. The field emission vs electric field plot should be an exponentially increasing curve if field emission is the current source. All of the samples shown in Fig. 13 demonstrate this exponential trait. The Ti-25/Fe-10 nm sample identified in Section 5.1 as the best overall CNT carpet sample clearly stands out as the best performer. The field emission test results are used to determine field emission properties or metrics for each sample. The turn-on electric field is the electric field strength required for a sample to reach a current density of $1 \mu \mathrm{A} / \mathrm{cm}^{2}$. As described above, the maximum current density achieved without damaging the CNT carpet and the corresponding electric field was also collected. The raw current vs voltage data was used to create Fowler-Nordheim Plots, $\ln \left(I / V^{2}\right)$ versus $1 / V$, for each sample as derived in Section 3.2. A linear Fowler-Nordheim plot is indicative of field emission. The Fowler-Nordheim plots for selected samples are contained in Fig. 14. Least squares linear regression was used to determine the slope, $m$, of the linear plots so the field enhancement factor, $\beta$, could be calculated using (7).

$$
\beta=\frac{b d \phi^{\frac{3}{2}}}{m}
$$

Where $b=6.83 \times 10^{7} \mathrm{eV}^{-3 / 2} \mathrm{Vcm}^{-1}, d=2.15 \times 10^{-4} \mathrm{~cm}$, and $\varphi=4.65 \mathrm{eV}$. All the field emission metrics are listed for each processed barrier/catalyst combination in Table 2.

As mentioned above, the Ti-25/Fe- $10 \mathrm{~nm}$ sample immediately jumps out as the best field emission source with the highest $\beta$, lowest turn-on electric field, and the highest total current density with the lowest electric field. Excellent field emission from the CNT carpet with the best qualities is a reassuring result. However, the improvement in field emission from adding the carbon buffer to the chrome barrier is astounding. The $\mathrm{Cr}-100 / \mathrm{Fe}-13 \mathrm{~nm}$ sample had extremely large diameter CNTs to the point of being considered carbon fiber more than carbon nanotubes. The field emission from these samples was also poor with a total current density of only $0.13 \mathrm{~mA} / \mathrm{cm}^{2}$.

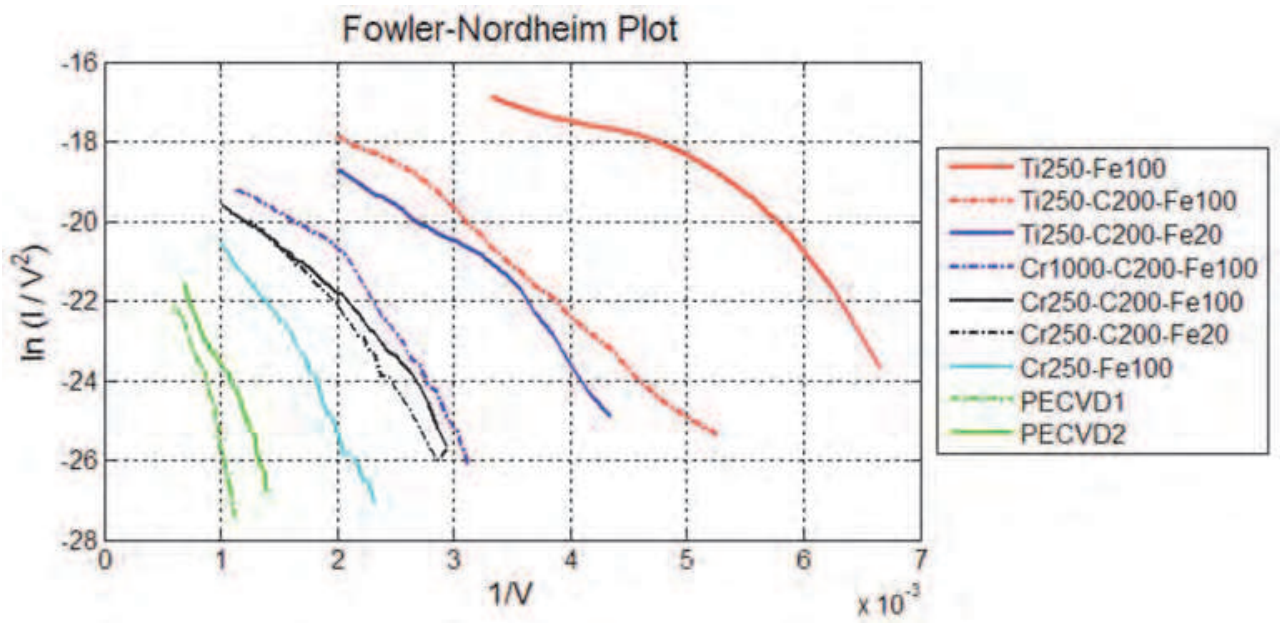

Fig. 14. Fowler-Nordheim plot for selected samples from Table 2. 
The Cr-100/C-20/Fe-10 nm sample on the other hand demonstrated a 30\% reduction in turn-on voltage and a 100x increase in maximum current density. The same is true for the $25 \mathrm{~nm}$ chrome barrier layers. While the addition of the carbon buffer did not have a significant effect on the characteristics of the CNT carpets showing moderate improvement in the Raman shift for the Cr-25/C-20/Fe-2 nm sample and a moderate degradation for the other sample, the improvements to the field emission properties are striking. Both samples saw a $20 \%$ reduction in turn-on electric field strength, $2.5 x$ and $6 x$ increases in maximum current density, and corresponding increases in the field enhancement factor. The carbon buffer layer clearly has a positive interaction with the chrome barrier that enhances field emission.

Much like the results on the CNT characteristics, the carbon buffer on the $25 \mathrm{~nm}$ titanium barrier layer degraded the field emission properties to include lower field enhancement factors, higher turn-on electric fields, and lower maximum current densities. The maximum current density between the Ti-25/Fe-2 nm and Ti-25/C-20/Fe-2 nm samples decreases from $9.25 \mathrm{~mA} / \mathrm{cm}^{2}$ to $2.63 \mathrm{~mA} / \mathrm{cm}^{2}$ at essentially the same electric field strength. As clearly as the carbon buffer improved the field emission properties of the chrome barrier samples, it degraded the field emission properties of the titanium barrier samples.

\begin{tabular}{|c|c|c|c|c|c|c|}
\hline \multicolumn{2}{|c|}{$\begin{array}{l}\text { Barrier Layer Type \& } \\
\text { Thickness (nm) }\end{array}$} & $\begin{array}{l}\text { Catalyst } \\
\text { (nm) }\end{array}$ & Beta & $\begin{array}{l}\text { Turn-on } \\
\text { E-field } \\
(\mathrm{V} / \mu \mathrm{m})\end{array}$ & $\begin{array}{c}\text { Max Current } \\
\text { Density } \\
\left(\mathrm{mA} / \mathrm{cm}^{2}\right)\end{array}$ & $\begin{array}{l}\text { Max E-field } \\
(\mathrm{V} / \mu \mathrm{m})\end{array}$ \\
\hline \multirow{4}{*}{ Chrome } & 100 & 13 & 4660 & 2.09 & 0.13 & 3.72 \\
\hline & 100 & 2 & \multicolumn{4}{|c|}{ No CNT Growth } \\
\hline & 25 & 10 & 3110 & 2 & 3.52 & 4.65 \\
\hline & 25 & 2 & 3630 & 1.95 & 0.34 & 4.19 \\
\hline \multirow{4}{*}{$\begin{array}{l}\text { Chrome \& } \\
\text { Carbon }\end{array}$} & $100 \& 20$ & 10 & 4870 & 1.49 & 12.30 & 4.14 \\
\hline & $100 \& 20$ & 2 & \multicolumn{4}{|c|}{ No CNT Growth } \\
\hline & $25 \& 20$ & 10 & 5430 & 1.58 & 8.71 & 4.65 \\
\hline & $25 \& 20$ & 2 & 4150 & 1.58 & 2.05 & 3.72 \\
\hline \multirow{4}{*}{ Titanium } & 100 & 10 & 6780 & 1.63 & 0.30 & 3.26 \\
\hline & 100 & 2 & 3130 & 2.23 & 3.99 & 5.12 \\
\hline & 25 & 10 & 8640 & 0.70 & 13.34 & 1.40 \\
\hline & 25 & 2 & 8020 & 0.93 & 9.25 & 2.37 \\
\hline \multirow{2}{*}{$\begin{array}{l}\text { Titanium \& } \\
\text { Carbon }\end{array}$} & $25 \& 20$ & 10 & 6070 & 0.88 & 12.94 & 2.33 \\
\hline & $25 \& 20$ & 2 & 6130 & 1.07 & 2.63 & 2.33 \\
\hline MPE-CVD \#1 & Ti 20 & Ni 10 & 2390 & 2.78 & 0.82 & 5.34 \\
\hline MPE-CVD \#2 & Ti 20 & Ni 10 & 3460 & 2.22 & 0.95 & 4.56 \\
\hline
\end{tabular}

Table 2. Field emission data for all combinations of barrier/catalyst samples. 
Field emission from the MPE-CVD samples does not compare well with the T-CVD samples. The MPE-CVD carbon nanotubes have a much higher areal density and are much shorter in length at $2 \mu \mathrm{m}$. Both of these factors increase screening effects reducing field emission. The controlled growth using MPE-CVD makes patterned and gated or triode configured field emission devices more feasible.

\section{Conclusion}

In this phase of a continuing research effort to develop CNT field emission devices, a study of the effects of $\mathrm{Ni}$ and Fe catalyst layers deposited on $\mathrm{Ti}$ and $\mathrm{Cr}$ diffusion barriers has been used to determine an optimum combination for effective field emission from CNTs produced via both T-CVD and MPE-CVD. As expected, thicker catalyst layers resulted in larger nano-particles after the anneal or pretreatment granulation process causing more amorphous carbon deposits as shown in SEM images. Raman spectroscopy was used to characterize the quality of the CNTs. The $\mathrm{G}^{\prime} / \mathrm{G}$ ratio was calculated as another possible metric that could relate Raman shift characteristics to field emission. Since no relationship was evident and there is no evidence that the $G^{\prime} / G$ ratio is helpful in determining CNT quality, the $G^{\prime} / G$ ratio was determined to be an unnecessary calculation for future endeavors.

This study showed that sputtered Fe catalyst films on thin barrier layers $(25 \mathrm{~nm})$ of $\mathrm{Ti}$ facilitate excellent CNT growth by T-CVD with superior CNT field emission properties, specifically, lower turn-on electric fields and much higher field emission current densities than any other combination of barrier and catalyst via either growth method. There was not a clear correlation between CNT quality as determined by Raman spectroscopy and CNT field emission. This is attributed to the effects CNT areal density has on field emission. When the areal density is extremely high, such as with the CNT carpets grown by MPECVD, screening effects impede field emission. When the CNT growth is sparse, the lack of field emission sources or CNTs impedes field emission. The best field emission was achieved by the Ti-25/Fe-100 sample which had both good Raman shift characteristics and qualitatively good or medium areal density.

The addition of a carbon buffer layer between chrome diffusion barriers and the catalyst layer showed some potential for improving the quality of CNT growth and increased field emission dramatically. The lack of CNT synthesis with thicker barrier layers was attributed to poor or at least different thermal properties of the thicker metallic barrier which impeded the granulation process resulting in incomplete separation of the catalyst nanoparticles shown through SEM micrographs. The lack of good quality CNT carpet growth from any of the $\mathrm{Cr}$ barrier samples, which are often used successfully in CNT synthesis, is a result of the significant impact different barrier layers have on the granulation and growth processes. Any changes to the barrier or catalyst will require the development of a specific growth recipe.

While the most successful field emission results were achieved with samples produced by TCVD, the MPE-CVD process allows better control and uniformity of the CNT lengths. The shorter length and high areal density of the MPE-CVD CNTs increasing screening effects is the primary cause of the lower field emission performance. However, this same control makes integration into electronic devices possible where the less controllable growth of the T-CVD process would potentially fail. Using the controllability of the MPE-CVD process gated CNT arrays are being fabricated to achieve field emission at lower voltages. 
Future research will focus on the interactions of the carbon buffer layer with the catalyst and barrier to determine the mechanism or process that drives the field emission improvements seen with the chrome barrier samples but not the titanium barrier samples. The T-CVD process will be refined to allow more control over the length and areal density of the CNTs for integration into gated devices.

\section{Acknowledgment}

The authors would like to thank the Air Force Research Laboratory (AFRL) Propulsion Directorate for the use of their resources and facilities, especially the MPE-CVD and T-CVD systems. The authors appreciate the technical support and access to cleanroom equipment provided by the AFRL Sensors branch RYD. As always the research would not be possible without the dedicated work of AFIT's own cleanroom support, Rick Patton and Rich Johnston.

Disclaimer: The views expressed in this article are those of the authors and do not reflect the official policy or position of the United States Air Force, Department of Defense, or the U.S. Government.

\section{References}

Amama, P., Pint, C., McJilton, L., Kim, S., Stach, E., Murray, T., et al. (2009). Role of water in super growth of single-walled carbon nanotube carpets. Nano Letters, Vol. 9, No. 1, pp. 44-49.

Bhushan, B. (Ed.). (2007). Springer Handbook of Nanotechnology, Springer, ISBN 3-54001218-4, Verlag, Germany.

Bonard, J., Kind, H., Stöckli, T., \& Nilsson, L. (2001). Field emission from carbon nanotubes: The first five years. Solid-State Electronics, Vol. 45, No. 6, pp. 893-914.

Chang, S., Lin, T., Li, T., \& Huang, S. (2008). Carbon nanotubes grown from nickel catalyst pretreated with H2/N2 plasma. Microelectronics Journal, Vol. 39, No. 12, pp. 15721575.

Chhoker, S., Srivastava, S., \& Vankar, V. (2007). Field emission properties of carbon nanostructures: A review, Proceedings of International Workshop on Physics of Semiconductor Devices, ISBN 978-1-4244-1728-5, Mumbai, December 2007.

Crossley, B., Kossler, M., Coutu Jr., R., Starman, L., \& Collins, P., (2010). Effects of hydrogen pretreatment on physical-vapor-deposited nickel catalyst for multi-walled carbon nanotube growth. Journal of Nanophotonics, Vol. 4, No. 1, pp. 049502-049502-6.

Crossley, B., Kossler, M., Starman, L., Coutu Jr., R., \& Collins, P., (2009). Optimization of carbon nanotube field emission arrays. Proceedings of COMSOL Conf., Vol. 6288, Boston, MA, October 2009.

DiLeo, R., Landi, B., \& Raffaelle, R., (2007). Purity assessment of multiwalled carbon nanotubes by Raman spectroscopy. Journal of Applied Physics, Vol. 101, No. 6, pp. 064307-064307-6.

Dresselhaus, M. S., Ado J., Hofman, M., Dresselhaus G., \& Saito, R., (2010). Perspectives on Carbon Nanotubes and Graphene Raman Spectroscopy, Nano Letters, Vol. 10, No. 3 , pp. 751-758.

Fursey, G. (2005). Field Emission in Vacuum Microelectronics. Kluwer Academic/Plenum Publishers, ISBN 0-3064-7450-6, New York. 
Garg, R., Kim, S., Hash, D., Gore, J., \& Fisher, T. (2008). Effects of feed gas composition and catalyst thickness on carbon nanotube and nanofiber synthesis by plasma enhanced chemical vapor deposition. Journal of Nanoscience and Nanotechnology, Vol. 8, No. 6, pp. 3068-3076.

Geckelar K., \& Rosenburg E. (2006). Functional Nanomaterials. American Scientific Publishers, ISBN 1-5888-3067-5, California.

Heer, W. A. d., Chatelain, A., \& Ugarte, D. (1995). A carbon nanotube field-emission electron source. Science, Vol. 270, No. 5239, pp. 1179-1180.

Iijima, S., (1991). Helical Microtubules of Graphitic Carbon, Nature, Vol 354, November 1991, pp. 56-58, ISSN 0028-0836.

Leonard, F. (2008). The Physics of Carbon Nanotube Devices. William Andrew, ISBN 978-08155-1573-9, Norwich, NY.

Nessim, G., Hart, A., Kim, J., Acquaviva, D., Oh, j., Morgan, C., et al. (2008). Tuning of vertically-aligned carbon nanotube diameter and areal density through catalyst pre-treatment. Nano Letters, Vol. 8, No. 11, pp. 3587-3593.

Nilsson, L., Groening, O., Emmenegger, C., Kuettel, O., Schaller, E., Schlapbach, L., et al. (2000). Scanning field emission from patterned carbon nanotube films. Applied Physics Letters, Vol. 76, No. 15, pp. 2071-2073.

O'Connell, M. (2006). Carbon Nanotubes: Properties and Applications. CRC Press, ISBN 0-84932748-2, Boca Raton, FL.

Paulini, J., Klein, T., \& Simon, G. (1993). Thermo-field Emission and the Nottingham Effect. Journal of Physics D: Applied Physics, Vol. 26, No. 8, 1310-1315.

Radushkevich, L., Lukyanovich, V. (1952). O structure ugleroda, obrazujucegosja pri termiceskom razlozenii okisi ugleroda na zeleznom kontakte. Zurn. Fisic. Chim., Vol. 26, pp. 88-95, Available from:<http://carbon.phys.msu.ru/publications/1952radushkevich-lukyanovich.pdf $>$.

Seelaboyina, R., Boddepalli, S., Noh, K., Jeon, M., \& Choi, W. (2008). Enhanced field emission from aligned multistage carbon nanotube emitter arrays. Nanotechnology, Vol. 19, No. 6, pp. 65605-65609.

Smith, R., \& Silva, S. (2009). Maximizing the electron field emission performance of carbon nanotube arrays. Applied Physics Letters, Vol. 94, No. 13, pp. 133104-133104-3.

Suh, J., Jeong, K., Lee, J., \& Han, H. (2002). Study of the field-screening effect of highly ordered carbon nanotube arrays. Applied Physics Letters, Vol. 80, No. 13, pp. 2392-2394.

Suzuki, S., Bower, C., Watanabe, Y., \& Zhou, O. (2000). Work functions and valence band states of pristine and cs-intercalated single-walled carbon nanotube bundles. Applied Physics Letters, Vol. 76, No. 26, pp. 4007-4009.

Tanaka, K., Yamabe, T., \& Fukui, K. (1999). The Science and Technology of Carbon Nanotubes. Elsevier, ISBN 0-0804-2696-4, New York, NY.

Terranova, M., Sessa, V., \& Rossi, M. (2006). The world of carbon nanotubes: An overview of CVD growth methodologies. Chemical Vapor Deposition, Vol. 12, No. 6, pp. 315-325.

Wong, Y., Kang, W., Davidson, J., Hofmeister, W., Wei, S., \& Huang, J. (2005). Device characterization of carbon nanotubes field emitters in diode and triode configurations. Diamond \& Related Materials, Vol. 14, No. 3, pp. 697-703.

Xu, Z., Bai, X., Wang, E., \& Wang, Z. (2005). Field emission of individual carbon nanotube with in situ tip image and real work function. Applied Physics Letters, Vol. 87, No. 16, pp. 163106-163106-3.

Zhu, W., Bower, C., Zhou, O., Kochanski, G., \& Jun, S. (1999). Large current density from carbon nanotube field emitters. Applied Physics Letters, Vol. 75, No. 6, pp. 873-875. 


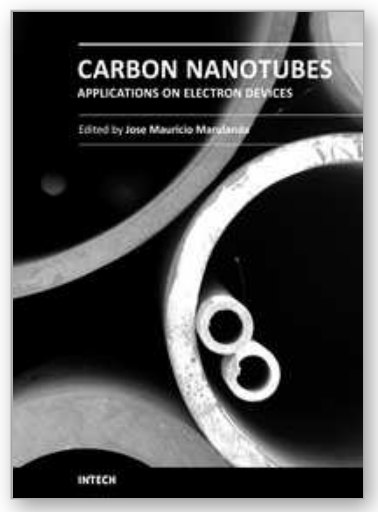

\section{Carbon Nanotubes Applications on Electron Devices}

Edited by Prof. Jose Mauricio Marulanda

ISBN 978-953-307-496-2

Hard cover, 556 pages

Publisher InTech

Published online 01, August, 2011

Published in print edition August, 2011

Carbon nanotubes (CNTs), discovered in 1991, have been a subject of intensive research for a wide range of applications. In the past decades, although carbon nanotubes have undergone massive research, considering the success of silicon, it has, nonetheless, been difficult to appreciate the potential influence of carbon nanotubes in current technology. The main objective of this book is therefore to give a wide variety of possible applications of carbon nanotubes in many industries related to electron device technology. This should allow the user to better appreciate the potential of these innovating nanometer sized materials. Readers of this book should have a good background on electron devices and semiconductor device physics as this book presents excellent results on possible device applications of carbon nanotubes. This book begins with an analysis on fabrication techniques, followed by a study on current models, and it presents a significant amount of work on different devices and applications available to current technology.

\section{How to reference}

In order to correctly reference this scholarly work, feel free to copy and paste the following:

Benjamin L. Crossley, Nathan E. Glauvitz, Betty T. Quinton, Ronald A. Coutu, Jr. and Peter J. Collins (2011). Characterizing Multi-Walled Carbon Nanotube Synthesis for Field Emission Applications, Carbon Nanotubes Applications on Electron Devices, Prof. Jose Mauricio Marulanda (Ed.), ISBN: 978-953-307-496-2, InTech, Available from: http://www.intechopen.com/books/carbon-nanotubes-applications-on-electrondevices/characterizing-multi-walled-carbon-nanotube-synthesis-for-field-emission-applications

\section{INTECH}

open science | open minds

\section{InTech Europe}

University Campus STeP Ri

Slavka Krautzeka 83/A

51000 Rijeka, Croatia

Phone: +385 (51) 770447

Fax: +385 (51) 686166

www.intechopen.com

\section{InTech China}

Unit 405, Office Block, Hotel Equatorial Shanghai

No.65, Yan An Road (West), Shanghai, 200040, China

中国上海市延安西路65号上海国际贵都大饭店办公楼405单元

Phone: +86-21-62489820

Fax: +86-21-62489821 
(C) 2011 The Author(s). Licensee IntechOpen. This chapter is distributed under the terms of the Creative Commons Attribution-NonCommercialShareAlike-3.0 License, which permits use, distribution and reproduction for non-commercial purposes, provided the original is properly cited and derivative works building on this content are distributed under the same license. 